\title{
Sarcopenia and Cortisol Among Older Nursing Home Residents: A Cross-Sectional Study
}

CRISTINA MARQUES DE ALMEIDA HOLANDA ( $\nabla$ cristinamahd@gmail.com )

Federal University of Rio Grande do Norte

PATRÍCIA VIDAL DE NEGREIROS NÓBREGA

Centro Universitário de João Pessoa

JOHN FONTENELE ARAÚJO

Federal University of Rio Grande do Norte

MARCIA REGINA PIUVEZAM

Federal University of Paraíba

HERMAN FERREIRA COSTA

Faculdade Nova Esperança

MAYLE ANDRADE MOREIRA

Universidade Federal do Ceará

ÁLVARO CAMPOS CAVALCANTI MACIEL

Federal University of Rio Grande do Norte

\section{Research Article}

Keywords: Sarcopenia, Nursing Homes, Aging, Hydrocortisone, Brazil.

Posted Date: October 12th, 2021

DOI: https://doi.org/10.21203/rs.3.rs-944122/v1

License: (c) (i) This work is licensed under a Creative Commons Attribution 4.0 International License.

Read Full License 


\section{Abstract}

Background: Recent studies indicate that the prevalence of sarcopenia among older adults living in longterm care facilities may be high. The pathophysiological mechanisms of sarcopenia are complex and interdependent. The role of cortisol deserves attention as a possible modulator in the genesis of sarcopenia. The objective this study is to analyze the relationship between sarcopenia and cortisol in older nursing home residents.

Method: A cross-sectional study conducted with 70 older people. Sarcopenia was assessed using the EWGSOP2 algorithm and cortisol by saliva collection. The T-test, Chi-squared test and ANOVA statistical analyzes were used $(p<0.05)$.

Results: A total of $17.1 \%$ of the older adults evaluated were considered sarcopenic. The related variables were older age $(p=0.001)$; lower body mass index $(p=0.008)$; lower measurements of brachial circumference $(p<0.001)$, waist $(p=0.011)$ and hips $(p=0.001)$. The cortisol levels were higher among the sarcopenic older adults for the three measures throughout the day $(p=0.02)$, as well as for the derived measures.

Conclusion: The higher salivary cortisol levels found in sarcopenic older nursing home residents help to understand the underlying mechanisms of sarcopenia and the healthcare of this public.

\section{Introduction}

Sarcopenia is currently understood as a progressive and generalized alteration of skeletal muscle, characterized by accelerated loss of muscle function and mass (1), with important health consequences. This disease is related to adverse health outcomes in older adults, and can be a predictor of mortality, functional decline, falls and hospitalization (2).

The prevalence of sarcopenia is substantial in most geriatric contexts, with emphasis on older adults living in long-term care facilities (LTCFs). Recent studies involving this theme indicate that the prevalence of sarcopenia among older adults living in LTCFs may be high (3-5), and shows variation according to the measuring instrument used (6).

Perkisas et al. (5) report that some clinical conditions which are predictors of admission to long-term care facilities, such as functional and cognitive decline, are related to the condition of sarcopenia among these older adults. Furthermore, this may be one of the reasons for the higher prevalence in this population. However, evidence from studies involving sarcopenia in an institutionalized environment is still limited because there are few studies which have addressed the topic of sarcopenia in this location and its associated factors (3). 
The pathophysiological mechanisms of sarcopenia are complex and interdependent. They involve an imbalance between the processes of muscle catabolism (proteolysis) and anabolism (protein synthesis). Age-associated loss of muscle mass occurs due to the gradual decline in muscle protein synthesis, combined with compromised control of proteolysis. Postprandial anabolic resistance is related to the aging process due to the deficiency in synthesizing proteins in response to several nutritional factors. In addition to these, other factors are associated with loss of muscle mass, such as lipotoxicity, insulin resistance, endocrine factors, oxidative stress, mitochondrial dysfunction and inflammation. Physical inactivity is another important aspect, as it can accelerate the process of muscle mass and strength loss (6).

Regarding the endocrine system, the role of cortisol deserves attention as a possible modulator in the genesis of sarcopenia. This hormone is a glucocorticoid produced by the hypothalamus-pituitary-adrenal (HPA) axis in response to challenging or threatening situations. The diurnal cortisol concentration circulating in the body provides information about the HPA axis activity and also serves as a negative feedback for the system itself to maintain its concentrations at a physiological level (7).

Cortisol is involved in several physiological systems, including the metabolic, immune system and the body's response to stress. Dysregulation of the HPA axis occurs in pathological situations such as Cushing's syndrome or even in situations of chronic physical or psychiatric stress. Excessive and continuous secretion of cortisol permeates muscle rupture, which may contribute to the onset of sarcopenia (8). However, there are still few studies involving the relationship between sarcopenia and changes in cortisol levels circulating in the body.

Thus, considering the sarcopenia condition among institutionalized older adults and the underlying mechanisms of possible neuroendocrine causes associated with sarcopenia, the present study aimed to analyze the relationship between sarcopenia and cortisol in LTCF older adults.

\section{Methods}

\subsection{Study design and ethical issues}

This is a cross-sectional observational study. The guidelines of the Strengthening the Reporting of Observational Studies in Epidemiology (STROBE) statement (9) were used. The Research Ethics Committee of the Health Sciences Center of the Federal University of Rio Grande do Norte - Brazil approved this study (protocol 201/09 CEP / UFRN). All methods were carried out in accordance with relevant guidelines and regulations. All participants signed the Informed Consent Form.

\subsection{Participants}

This study was conducted in the city of João Pessoa in northeastern Brazil. Six LTCFs were for the study were identified from municipal health agencies. The total population consisted of 374 older adults. Participants in this research were the same as those included in previous studies $(10,11)$ 
The following parameters were used to calculate the sample based on cross-sectional studies: $20.0 \%$ margin of error, non-response rate of $20.0 \%$, finite population size (374 older adult) and estimated prevalence of sarcopenia found in the literature with regard to the target age group of $17 \%$ (12). A further $15 \%$ was added to the sample number due to possible losses. The study was carried out with a total sample of 70 subjects. Sampling was done by simple random probability.

Inclusion criteria were: being 60 years of age or older, having a minimum score of 17 points on the MiniMental State Examination (MMSE) (13), not having an unfavorable clinical condition for performing the specific tests. The older adults who were excluded from the data collection gave up on participating in the research, presented an unfavorable clinical condition during the data collection, or were unable to answer the essential research questions $(10,11)$.

\subsection{Measurements}

\subsubsection{Sarcopenia}

Sarcopenia was classified according to the updated consensus of European Working Group on Sarcopenia in Older People (EWGSOP2). Participants with low muscle strength are identified as people likely to present sarcopenia. It is analyzed whether the individual has low muscle quality or quantity to confirm the diagnosis. Finally, sarcopenia is considered severe if the older adults also presents low physical performance (14).

Following the algorithm for assessing sarcopenia proposed by the EWGSOP2, handgrip strength was used to verify muscle strength with the following cut-off points for sarcopenia: $<27 \mathrm{~kg}$ for men and $<16$ $\mathrm{kg}$ for women $(14,15)$.

Muscle mass was estimated by appendicular skeletal muscle mass (ASM) using the following anthropometric prediction equation (PE) $(16,17)$ : ASM $(P E)=10.05+0.35$ (weight) -0.62 (BMI) -0.02 (age) + 5.10 (if male). Body weight was measured in kilograms $(\mathrm{kg})$ and height was measured in meters $(\mathrm{m})$. The body mass index (BMI) was calculated from the measured height and weight $\left(\mathrm{kg} / \mathrm{m}^{2}\right)$. After estimating the values, we adjusted the ASM by height squared to create the skeletal muscle mass index $\left(\mathrm{SMI}=\mathrm{ASM} /\right.$ height $\left.^{2}\right)(17)$. Following previous studies (18-20), the cut-off for low muscle mass used was based on the $20 \%$ lowest percentile of the population distribution, representing a SMl of $\leq 4.91 \mathrm{~kg} / \mathrm{m}^{2}$ for women and $\leq 7.47 \mathrm{~kg} / \mathrm{m}^{2}$ for men. We chose to use the validated prediction equation in place of conventional techniques such as bioimpedance or DEXA (16) due to the operational difficulty of older adults to follow the preparation protocol for taking body composition measurements.

Physical performance was assessed through gait speed. Gait speed $\leq 0.8 \mathrm{~m} / \mathrm{s}$ was considered as a cutoff point for low performance and therefore classified older adults as having severe sarcopenia $(14,21)$.

\subsubsection{Cortisol}


Cortisol levels can be measured in body fluids present in blood, urine or saliva (22). The salivary cortisol measurement was chosen according to the procedures previously adopted by Holanda et al (10). Salivary cortisol is an effective method to assess adrenal cortex functioning and has been widely used in studies evaluating the activity of this gland (23). The salivary cortisol collection procedure is simple, safe, replicable, does not cause pain or discomfort, and does not require more qualified training for collection. Furthermore, the cortisol in saliva is stable at room temperature, which facilitates its collection and transport (22).

Biochemical cortisol analysis was performed at a laboratory using enzyme immunoassay with photometric analysis. The procedures followed the protocol established by the Enzyme Linked Immunosorbent Assay (ELISA) kit for high-sensitivity salivary cortisol analysis (Salimetrics, LLC). The samples were analyzed in duplicate, optical density was read on a standard plate reader at $450 \mathrm{~nm}$ (BioRad Laboratories, model 550) and the concentration of each replicate was established from a standard curve. The minimum cortisol concentration able to be detected was $0.003 \mu \mathrm{g} / \mathrm{dL}$. The final cortisol values obtained were transformed by logarithmic interpolation and presented in the $\mathrm{nmol} / \mathrm{L}$ unit.

Saliva samples were collected three times throughout the day for each subject. The first collection was between 6 and 7 a.m., the second collection between 11 a.m. and 12 p.m., and the third collection between 5 and 6 p.m. It was then possible to analyze other parameters from the cortisol measurements, including the daily cortisol average (the sum of the three measurements divided by 3 ), the cortisol amplitude (the first measurement minus the third) and the rate of decline in cortisol (obtained by the linear regression curve for the 1st, 2nd and 3rd cortisol measurements) (10).

\subsubsection{Demographic and health-related information}

Information on sociodemographic aspects and those related to physical health was collected through a questionnaire constructed by the researchers. Age, sex, educational level, marital status, color/race, and time living in the institution comprised the sociodemographic data. Physical health aspects included information on the presence of self-information of chronic pathologies, depressive symptoms, selfperception of health, occurrence of falls, hospitalization and cognitive function through application of the $\operatorname{MMSE}(10,11)$.

\subsection{Procedures}

Data collection procedures can be observed with more details in Holanda et al (10). Pilot study subjects were not included in the outcome analyses. Each LTCF provided a list of registered older adults. The number of interviewed subjects from each institution was defined proportional to its total population. A simple draw was performed to sample the subjects. Subjects who met the predefined criteria and gave their free and informed consent were included.

The collections were carried out in two days inside the institution's rooms. On the first day, the questionnaire with sociodemographic and physical health data was answered. It started with the interview and then with the physical tests. Some data from the questionnaire were completed by the team 
of professionals from the institutions. The gait speed was calculated on a straight course of 4.6 meters. Three attempts were made and the final speed was calculated by the average of the speeds. The time was recorded by stopwatch in milliseconds. Weight and height were measured with a Filizola series 3.134 scale and a fiberglass measuring tape, respectively. Handgrip strength was calculated with the aid of a dynamometer (JAMAR J00105 portable hydraulic dynamometer). The subject used their dominant hand and the average grip strength was calculated from three attempts. For this test, the subject was positioned in a sitting position, with their arm at the side of their body, elbow at 90 degrees and forearm and wrist in a neutral position.

The second day was dedicated to collecting salivary cortisol. The subjects were approached at three different times, always before meals, according to the data reported in the previous sections. The subjects were instructed that they could not eat, brush their teeth, smoke or drink alcoholic beverages $2 \mathrm{~h}$ before each collection (24). Saliva was collected by passive drip into $1.5 \mathrm{ml}$ Ependorff tubes. After collection, saliva samples were transported in refrigerated bags and stored in a freezer at $-20^{\circ} \mathrm{C}$.

\subsection{Statistical analysis}

The SPSS (Statistical Package for the Social Sciences), version 20 computer program was used to create the database and perform the statistical analyzes. The means, standard deviations, relative frequencies and absolute frequencies were calculated for descriptive analysis. Data were organized and grouped according to sarcopenia status. The normality calculation was determined by the Kolmogorov-Smirnov (K-S) test. After confirming normality, bivariate analyzes were carried out by applying the T-test (for quantitative variables) and the chi-squared test (for categorical variables). In the analysis of the cortisol curves according to sarcopenia, the differences between the means of the measurements in each group were verified by analysis of variance (ANOVA) for repeated measurements, with post hoc Tukey's test. The cortisol decline rate per hour was calculated with the help of the GraphPad Prism version 5.00 program. Cortisol measurements in nmol were also calculated by this Program. A 95\% confidence interval $(\mathrm{Cl})$ and $p<0.05$ was established for all statistical analyses.

\section{Results}

The sample consisted of 70 subjects, 25 men (35.7\%) and 45 women (64.3\%), with a mean age of 77.78 ( \pm 7.87 ) years. Regarding the self-report of chronic diseases, the most prevalent conditions were hypertension (61.4\%), arthropathies (50\%) and the presence of depressive symptoms (32.9\%). Regarding the presence of sarcopenia, 12 individuals (17.1\%) were considered sarcopenic according to previously established criteria. The sociodemographic and physical health aspects were not statistically different for individuals who presented or who did not present sarcopenia. However, sarcopenic individuals were older than those who did not present sarcopenia $(p=0.001)$. Anthropometric measurements such as arm, waist and hip circumferences were also different between groups (Table 1).

Higher rates regarding cortisol measurements were observed in the first measurement, with a decline throughout the day. The mean cortisol for the first measurement was $3.51( \pm 1.98) \mathrm{nmol} / \mathrm{L}$, followed by 
the second measurement $2.33( \pm 1.31) \mathrm{nmol} / \mathrm{L}$ and the third measurement $1.46( \pm 0.86) \mathrm{nmol} / \mathrm{L}$. Mean cortisol concentrations throughout the day are statistically different $(1 \mathrm{st}>2 \mathrm{nd}>3 \mathrm{rd})(\mathrm{p}<0.001)$.

Considering the main objective of the present study, Figure 1 shows the diurnal salivary cortisol curves considering the three measurements throughout the day between the older adults with presence and absence of sarcopenia. As we can observe, there were significant differences for all three measurements $(p=0.020)$ between the groups. The cortisol levels were higher between the older adults presenting sarcopenia for the three measures throughout the day, including the higher levels at the end of the day. Significant differences were observed regarding the repeated measurements ANOVA between the three measurements ( $1 \mathrm{st}>2 \mathrm{nd}>3 \mathrm{rd}$ ) for both sarcopenic older adults as well as for those who were not sarcopenic. Furthermore, we observed significant differences regarding cortisol amplitude $(p=0.011)$ and decline rate $(p=0.011)$ (Table 2$)$, with higher values being found between sarcopenic individuals.

\section{Table 1}

Sociodemographic, anthropometric and health data according to the sarcopenia classification 


\begin{tabular}{|c|c|c|c|c|c|c|c|}
\hline & \multicolumn{7}{|c|}{ SARCOPENIA } \\
\hline & \multicolumn{2}{|l|}{ Yes } & \multicolumn{2}{|l|}{ No } & \multicolumn{3}{|l|}{ Total } \\
\hline & $\mathbf{n}$ & $\%$ & $\mathbf{n}$ & $\%$ & $\mathbf{n}$ & $\%$ & $\mathbf{p}$ \\
\hline \multicolumn{8}{|l|}{ SEX } \\
\hline Male & 5 & 7.1 & 20 & 28.6 & 25 & 35.7 & \multirow[t]{2}{*}{0.744} \\
\hline Female & 7 & 10 & 38 & 54.3 & 45 & 64.3 & \\
\hline$A G E^{*}$ & 84.16 & \pm 6.35 & 76.10 & 7.48 & 77.48 & 7.87 & 0.001 \\
\hline EDUCATION* & 4.33 & \pm 5.03 & 4.81 & 4.22 & 4.72 & 4.33 & 0.731 \\
\hline TIME IN INSTITUTION* & 5.25 & \pm 12.84 & 4.84 & 5.47 & 4.91 & 7.15 & 0.860 \\
\hline MEEM (points) ${ }^{*}$ & 21.08 & \pm 3.98 & 23.29 & 3.66 & 22.91 & 3.78 & 0.65 \\
\hline \multicolumn{8}{|l|}{ SELF-RATED HEALTH } \\
\hline Satisfactory & 7 & 10 & 33 & 47.1 & 40 & 57.1 & \multirow[t]{2}{*}{0.927} \\
\hline Unsatisfactory & 5 & 7.1 & 25 & 35.7 & 30 & 42.9 & \\
\hline NUMBER OF MEDICATIONS * & 2.50 & \pm 1.83 & 3.46 & 2.63 & 3.30 & 2.53 & 0.232 \\
\hline $\mathrm{BMI}$ * & 22.22 & \pm 6.55 & 27.10 & 5.39 & 26.27 & 5.85 & 0.008 \\
\hline ARM CIRCUMFERENCE * & 21.24 & \pm 3.47 & 26.35 & 3.82 & 25.48 & 4.21 & $<0.001$ \\
\hline WAIST CIRCUMERENCE * & 85.50 & \pm 11.63 & 95.21 & 11.65 & 93.55 & 12.14 & 0.011 \\
\hline HIP CIRCUMFERENCE * & 89.03 & \pm 9.53 & 100.85 & 10.44 & 98.82 & 11.17 & 0.001 \\
\hline \multicolumn{8}{|c|}{ Note. Significant values are in bold ( $P$ value $<0.05)$. } \\
\hline
\end{tabular}

\section{Table 2}

Cortisol level means according to sarcopenia. 


\begin{tabular}{|llll|}
\hline & \multicolumn{2}{l|}{ Sarcopenia } & \\
\cline { 2 - 4 } & No & Yes & \\
\cline { 2 - 4 } & Mean (SD) & Mean (SD) & P-value \\
\hline Cortisol mean & $2.15( \pm 0.90)$ & $3.46( \pm 1.41)$ & 0.009 \\
\hline Cortisol 1 & $3.21( \pm 1.66)$ & $4.92( \pm 2.77)$ & 0.061 \\
\hline Cortisol 2 & $2.07( \pm 1.13)$ & $3.56( \pm 1.46)$ & $<0.001$ \\
\hline Cortisol 3 & $1.36( \pm 0.85)$ & $1.91( \pm 0.81)$ & 0.045 \\
\hline Cortisol amplitude & $1.97( \pm 1.19)$ & $3.13( \pm 1.39)$ & 0.011 \\
\hline Decline rate & $-0.19( \pm 0.11)$ & $-0.31( \pm 0.13)$ & 0.011 \\
\hline Note. Significant values are in bold $(\mathrm{P}$ value $<0.05)$. Cortisol parameters $(\mathrm{nmol} / \mathrm{L})$. \\
\hline
\end{tabular}

Regarding the degree of severity of sarcopenia, all sarcopenic older adults in the sample were considered to have severe sarcopenia, since they obtained gait speed $\geq 0.8 \mathrm{~m} / \mathrm{s}$ during the physical performance test (data not shown in the table).

\section{Discussion}

The main objective of this study was to verify the relationship between sarcopenia and salivary cortisol levels in older adults LTCF residents. There is wide variability in the sarcopenia prevalence reported in the literature (25). The sarcopenia prevalence found in the sample (17.1\%) is in agreement with another Brazilian study which estimated a prevalence of $17 \%$ through a systematic review for the country (12). However, these values are closer to the lower limits of other studies published in the international literature for older adults living in LTCFs, such as in the studies by Shen et al.(3) and Rodríguez-Rejón et al. (26), who found a variation of 22 to $85 \%$ and 17.7 to $73.3 \%$, respectively. It is important to highlight that the findings on sarcopenia prevalence are intrinsically related to the assessment methods and instruments, the cut-off points used, the inclusion and exclusion criteria of the sample, as well as linked to the population characteristics of each sample.

The age variable regarding demographic information is noteworthy, as the sarcopenic older adults also had the highest mean age. It is known that the aging process is accompanied by a tendency to decrease muscle mass and strength, and that this process can be modulated by genetics and lifestyle (14). There is strong evidence that the incidence of sarcopenia increases with age (1) and several studies also corroborate this factor among institutionalized older adults $(4,27,28)$. However, through a systematic review that investigated the prevalence and factors associated with sarcopenia in LTCFs, Shen et al.(3) found that age still maintains an inconsistent association with sarcopenia and that new studies are necessary to assess this relationship. 
Mean arm, hip and waist circumferences, as well as BMI itself, were also lower in the sarcopenic older adult group. This difference may be related to the nutritional status of the subjects, as several studies associate poor nutrition with the presence of sarcopenia (27-30). In addition, it is noteworthy that the $\mathrm{BMI}$ measurements are also linked to the adopted skeletal muscle mass prediction calculations. In the present investigation, with the exception of age, no relationship was found between sociodemographic variables and specific chronic health conditions with sarcopenia, although some studies indicate that female gender (27) and cognitive dysfunctions (28), for example, may be associated.

The relationship found between sarcopenia and salivary cortisol, the main objective of this study, can be determined by the difference between the cortisol measurements verified throughout the day, in which the sarcopenic older adult group had higher values than the non-sarcopenic older adult group. In addition to the differences between the values of the first, second and third measurements, the mean value, the decline rate and the amplitude values derived from the cortisol measurements were also higher in the sarcopenic older adult group.

Waters et al. (31) found higher salivary cortisol concentrations in a group of sarcopenic older adults when compared to older adults classified in other body compositions (normal, obese and obese-sarcopenic). These results were expressed both by the cortisol peak after breakfast, as well as by the area under the cortisol curve (AUC) performed during the day.

Yanagita et al. (8) analyzed (among other variables) the balance between the cortisol and dehydroepiandrosterone sulfate (DHEA-S) hormones in sarcopenic and non-sarcopenic older adult patients with Type 2 Diabetes Mellitus. They found that there were significantly higher cortisol values and lower DHEA-S values in the sarcopenic group, which raised the cortisol/DHEA-S ratio. The study showed that a cortisol/DHEA-S ratio $\geq 0.2$ was the strongest risk factor for sarcopenia in older adult diabetic patients. Furthermore, they observed that the more severe the sarcopenia, the higher the circulating cortisol levels and lower DHEA-S levels, indicating a probable influence of adrenal hormones on the pathophysiology of sarcopenia. Although the present study did not assess the function of DHEA-S, it was possible to carry out a comparison of cortisol levels alone.

Westbury et al. (32) investigated the association of inflammation markers, including cortisol, with the components of muscle mass, strength and function, in addition to the verification of sarcopenia using the algorithm proposed by the EWGSOP through a longitudinal study among community-dwelling older adults. The authors found that a higher cortisol level was considered a predictor of a decrease in the subjects' appendicular lean mass after 10.8 years of follow-up.

Another study compared patients, men and women, with adrenal incidentaloma (Al) classified into two groups: non-functioning Al and those with subclinical hypercotisolemia. The results showed that the excess, even if slight, of cortisol present in the second group was associated with lower skeletal muscle mass among female patients (33). This study did not collect enough data for the classification needed for sarcopenia. 
These findings reinforce the relationship of the hormone cortisol with sarcopenia and/or with the musculoskeletal system, which is intrinsically related to the pathophysiology of sarcopenia. However, unlike the present study, most of the articles mentioned analyzed cortisol through single collections. This increases the methodological problems of diurnal cortisol assessment, as it is a hormone which undergoes several changes in concentrations throughout the day (7). In addition, the studies used different methods to assess sarcopenia, which also makes it difficult to compare the results.

Cortisol-derived measures, such as cortisol decline index and amplitude, represent diurnal variation in cortisol, and lower values indicate lesser resilience of the HPA axis (34). Both were higher in sarcopenic older adults, indicating a greater drop in cortisol per hour. However, despite the greater descending slope of the cortisol curve, the amount of cortisol in sarcopenic older adults remained higher throughout the day than in non-sarcopenic older adults.

An important fact is that all the older adults identified as sarcopenic in this study were classified as having severe sarcopenia. It is possible that most older adult LTCF residents are considered frail or prefrail (10) and therefore received the most severe classification for sarcopenia.

A higher cortisol concentration in sarcopenic older adult LTCF residents suggests that these individuals may be more exposed to a greater degree of stress when compared to institutionalized older adults who do not have sarcopenia. Cortisol is considered an important marker of physiological stress. It is released by the adrenal glands from the activation of the HPA axis, and is of substantial importance, since its concentrations interfere in various systems of the body. The normal aging process is associated with changes in the endocrine system with consequent hormonal changes, which include the gradual and sustained increase in glucocorticoids (35).

Sarcopenia is characterized by loss of muscle mass and function, is often associated with age, and can be considered a complex geriatric syndrome whose pathophysiology is not very clear. The systems involved include neural and hormonal regulation, in addition to muscle degradation (36). Tournadre et al. (6) describes that the muscle is a contractile organ which enables the human body to move. In addition, it plays other important roles as it is essential for metabolic homeostasis. Muscle can act in glucose uptake, glycogen storage, lipid oxidation, amino acid release, energy production, as well as serving as an amino acid reservoir for immunocompetent cells.

In an attempt to deepen the understanding of the pathophysiological processes involved in the diagnosis and treatment of sarcopenia, some studies have emphasized biochemical analyzes seeking to find biomarkers which may be associated with sarcopenia $(37,38)$. In this sense, the present study can contribute by reinforcing the understanding of the cortisol relationship in the complex underlying mechanism of sarcopenia.

Some considerations about the study limitations permeate the fact that more cortisol collections, and in more than one day, could improve understanding of the cortisol concentration curve. In addition, the assessment of sarcopenia was based on the EWGSOP2 algorithm, which has European populations as 
baseline studies, and which therefore is subject to the need to adapt to the Brazilian population. Regarding the evaluation of the item amount of muscle present in the algorithm used for sarcopenia, a prediction equation validated by the impossibility of applying a gold standard measure of evaluation was used. However, it is worth noting that these limitations were mainly due to the difficulty of using more complex equipment and routines in the LTCF, in addition to all the restrictions (physical, sanitary, temporal, etc.) imposed by them. Finally, the inclusion criteria used which limited the participation of older adults with severe cognitive impairments or severe physical disabilities, may have excluded the participation of older adults who had sarcopenia, reducing the prevalence found for this condition in the group of older adults studied at the LTCF.

\section{Conclusion}

Finally, the present study observed that higher amounts of salivary cortisol are related to the condition of sarcopenia in older adults living in LTCFs. New studies which clarify the cause and effect relationship and that deepen the knowledge about biomarkers for sarcopenia are needed.

\section{Declarations}

Ethics approval and consent to participate: The research project was submitted for consideration and approval to the Research Ethics Committee of the Center for Health Sciences at Federal University of Rio Grande do Norte, Brazil (protocol no 201/09 CEP/UFRN). All participants gave informed consent.

Consent for publication: Not applicable.

Availability of data and materials: The datasets used and/or analysed during the current study are available from the corresponding author on reasonable request.

Competing interests: The authors declare that they have no competing interests.

Funding: This study was financed in part by the Coordenação de Aperfeiçoamento de Pessoal de Nível Superior - Brasil (CAPES) - Finance Code 001.

Authors' contributions: CMAH participated in Conceptualization, Methodology, Formal analysis, Investigation, Writing - Original Draft, Writing - Review \& Editing; PVNN participated in Investigation, Writing - Original Draft; JFA participated in Writing - Review \& Editing, Supervision; MRP participated in Writing - Review \& Editing, Resources; HFC participated in Writing - Review \& Editing, Formal analysis; MAM participated in Formal analysis, Writing - Original Draft, Writing - Review \& Editing, Supervision; ACCM participated in Conceptualization, Methodology, Formal analysis, Writing - Review \& Editing, Supervision, Funding acquisition. All authors read and approved the final manuscript.

Acknowledgements: Not applicable 


\section{References}

1. Cruz-Jentoft AJ, Sayer AA. Sarcopenia. Lancet. 2019;393(10191):2636-46.

2. Beaudart C, Zaaria M, Pasleau F, Reginster JY, Bruyère 0 . Health outcomes of sarcopenia: A systematic review and meta-analysis. PLoS One. 2017;12(1):1-16.

3. Shen Y, Chen J, Chen X, Hou L, Lin X, Yang M. Prevalence and Associated Factors of Sarcopenia in Nursing Home Residents: A Systematic Review and Meta-analysis. J Am Med Dir Assoc [Internet]. 2018;1-9. Available from: https://doi.org/10.1016/j.jamda.2018.09.012

4. Bravo-José P, Moreno E, Espert M, Romeu M, Martínez P, Navarro C. Clinical Nutrition ESPEN Prevalence of sarcopenia and associated factors in institutionalised older adult patients Concepci 0. Clin Nutr ESPEN. 2018;1-7.

5. Perkisas S, De Cock AM, Vandewoude M, Verhoeven V. Prevalence of sarcopenia and 9-year mortality in nursing home residents. Aging Clin Exp Res [Internet]. 2019;31(7):951-9. Available from: http://dx.doi.org/10.1007/s40520-018-1038-2

6. Tournadre A, Vial G, Capel F, Soubrier M, Boirie Y. Sarcopenia. Jt Bone Spine. 2018;16(2):1-6.

7. Gaffey AE, Bergeman CS, Clark LA, Wirth MM. Aging and the HPA axis: Stress and resilience in older adults. Neurosci Biobehav Rev [Internet]. 2016;68:928-45. Available from:

http://dx.doi.org/10.1016/j.neubiorev.2016.05.036

8. Yanagita I, Fujihara Y, Kitajima Y, Tajima M, Honda M, Kawajiri T, et al. A High Serum Cortisol / DHEAS Ratio Is a Risk Factor for Sarcopenia in Elderly Diabetic Patients. J Endocr Soc. 2019;3(March):801-13.

9. Malta M, Cardoso LO, Bastos FI, Magnanini MMF, Silva CMF da. Iniciativa STROBE: subsídios para a comunicação de estudos observacionais STROBE initiative : guidelines on. 2010;44(3):559-65.

10. Holanda CM de A, Guerra RO, Nóbrega PV de N, Costa HF, Piuvezam MR, Maciel ÁcC. Salivary cortisol and frailty syndrome in elderly residents of long-stay institutions: A cross-sectional study. Arch Gerontol Geriatr. 2012;54(2):146-51.

11. Nóbrega PV de N, Maciel ÁCC, de Almeida Holanda CM, Oliveira Guerra R, Araújo JF. Sleep and frailty syndrome in elderly residents of long-stay institutions: A cross-sectional study. Geriatr Gerontol Int. 2014;14(3):605-12.

12. Diz JBM, Leopoldino AAO, Moreira BDS, Henschke N, Dias RC, Pereira LSM, et al. Prevalence of sarcopenia in older Brazilians: A systematic review and meta-analysis. Geriatr Gerontol Int. 2016;112.

13. Brucki SMD, Nitrini R, Caramelli P, Bertolucci PHF, Okamoto IH. SUGESTÕES PARA O USO DO MINIEXAME DO ESTADO MENTAL NO BRASIL. Arq Neuropsiquiatr. 2003;61(3-B):777-81.

14. Cruz-Jentoft AJ, Bahat G, Bauer J, Boirie $Y$, Bruyère 0 , Cederholm T, et al. Sarcopenia: revised European consensus on de fi nition and diagnosis. Age Ageing. 2018;(1):1-16.

15. Dodds RM, Syddall HE, Cooper R, Benzeval M, Deary IJ, Dennison EM, et al. Grip Strength across the Life Course: Normative Data from Twelve British Studies. PLoS One. 2014;9(12):1-15. 
16. Visvanathan R, Yu S, Field J, Chapman I, Adams R, Wittert G, et al. APPENDICULAR SKELETAL MUSCLE MASS: DEVELOPMENT AND VALIDATION OF ANTHROPOMETRIC PREDICTION EQUATIONS. J Frailty Aging. 2012;1(4):147-51.

17. Yu S, Appleton S, Chapman I, Adams R, Wittert G, Visvanathan T, et al. An Anthropometric Prediction Equation for Appendicular Skeletal Muscle Mass in Combination With a Measure of Muscle Function to Screen for Sarcopenia in Primary and Aged Care. JAMDA [Internet]. 2015;16(1):25-30. Available from: http://dx.doi.org/10.1016/j.jamda.2014.06.018

18. Newman AB, Kupelian ÃV, Visser ÃM, Simonsick E, Goodpaster B, Nevitt ÃM, et al. Lower Extremity Function. J Am Geriatr Soc. 2003;51:1602-9.

19. Delmonico MJ, Harris ÃTB, Lee J, Visser M, Nevitt M, Kritchevsky SB, et al. Alternative Definitions of Sarcopenia, Lower Extremity Performance, and Functional Impairment whit Aging in Older Men and Women. Am Geriatr Soc. 2007;55(5):769-74.

20. Pagotto V, Silveira EA. Methods, Diagnostic Criteria, Cutoff Points, and Prevalence of Sarcopenia among Older People. Sci World J. 2014;2014.

21. Jentoft AJC, Baeyens JP, Bauer JM, Boirie Y, Cederholm T, Landi F, et al. Sarcopenia: consenso europeo sobre su definición y diagnóstico Informe del Grupo europeo de trabajo sobre la sarcopenia en personas de edad avanzada. 2010;44(0):412-23.

22. Bozovic D, Racic M, Ivkovic N. Salivary Cortisol Levels as a Biological Marker of Stress Reaction. Med Arch. 2013;67(05):371-4.

23. Speer KE, Semple S, Naumovski N, Cunha NMD, Mckune AJ. Neurobiology of Stress HPA axis function and diurnal cortisol in post-traumatic stress disorder: A systematic review. Neurobiol Stress [Internet]. 2019;11(December 2018):100180. Available from:

https://doi.org/10.1016/j.ynstr.2019.100180

24. Kokavec A, Lindner AJ, Ryan JE, Crowe SF. Pharmacology, Biochemistry and Behavior Ingesting alcohol prior to food can alter the activity of the hypothalamic-pituitary-adrenal axis. Pharmacol Biochem Behav [Internet]. 2009;93(2):170-6. Available from:

http://dx.doi.org/10.1016/j.pbb.2009.05.004

25. Dhillon RJS, Hasni S. P a t h o g e n e s i s an d M a n a g e m e n t of $S$ a rco p e $n$ i a. Clin Geriatr Med. 2017;33(3):17-26.

26. Rodríguez-Rejón Al, Ruiz-López MD, Wanden-Berghe C, Artacho R. Prevalence and Diagnosis of Sarcopenia in Residential Facilities: A Systematic Review. Adv Nutr. 2019;10(1):51-8.

27. Yap SF, Boo NY, Pramod DS, Thaw Z, Liew SF, Woo LF, et al. Risk Factors Associated with Sarcopenia Among Independently Mobile, Institutionalised Older People in the Klang Valley of Malaysia : A Cross-Sectional Study. Malaysian J Med Sci. 2020;27(2):120-8.

28. Yalcin A, Aras S, Atmis V, Cengiz OK, Varli M, Cinar E, et al. Sarcopenia prevalence and factors associated with sarcopenia in older people living in a nursing home in Ankara Turkey. Geriatr Gerontol Int. 2015;1-8. 
29. Cruz-Jentoft AJ, Kiesswetter E, Drey M, Sieber CC. Nutrition, frailty, and sarcopenia. Aging Clin Exp Res. 2017;29(1):43-8.

30. Yang LJ, Wu GH, Yang YL, Wu YH, Zhang L, Wang MH, et al. Nutrition, physical exercise, and the prevalence of sarcopenia in elderly residents in nursing homes in China. Med Sci Monit. 2019;25:4390-9.

31. Waters DL, Qualls CR, Dorin RI, Veldhuis JD, Baumgartner RN. Altered growth hormone, cortisol, and leptin secretion in healthy elderly persons with sarcopenia and mixed body composition phenotypes. Journals Gerontol - Ser A Biol Sci Med Sci. 2008;63(5):536-41.

32. Westbury LD, Fuggle NR, Syddall HE, Duggal NA, Shaw SC, Maslin K, et al. Relationships Between Markers of Inflammation and Muscle Mass, Strength and Function: Findings from the Hertfordshire Cohort Study. Calcif Tissue Int [Internet]. 2018 Mar 3 [cited 2020 Sep 17];102(3):287-95. Available from: http://link.springer.com/10.1007/s00223-017-0354-4

33. Kim HJ, Kwak MK, Ahn SH, Kim H, Cho YY, Suh S, et al. Alteration in skeletal muscle mass in women with subclinical hypercortisolism. Endocrine [Internet]. 2018; Available from:

http://dx.doi.org/10.1007/s12020-018-1598-0

34. Varadhan R, Walston J, Cappola AR, Carlson MC, Wand GS, Fried LP. Higher Levels and Blunted Diurnal Variation of Cortisol in Frail Older Women. J Gerontol. 2008;63(2):190-5.

35. Andreas Y, Tsioutis C, Agapidaki E, Zafeiri M, Agouridis AP, Ntourakis D, et al. Adrenal Aging and Its Implications on Stress Responsiveness in Humans. Front Endocrinol (Lausanne). 2019;10(54).

36. Cruz-Jentoft AJ, Landi F, Schneider SM, Zúñiga C, Arai H, Boirie Y, et al. Prevalence of and interventions for sarcopenia in ageing adults: a systematic review. Report of the International Sarcopenia Initiative (EWGSOP and IWGS). Age Ageing. 2014;0:1-12.

37. Rodrigues-Mañas L, Carvalho IA de, Bhasin S, Bischoff-Ferrari HA, Casari M, Evans W, et al. ICFSR TASK FORCE PERSPECTIVE ON BIOMARKERS FOR SARCOPENIA AND FRAILTY. J Frailty Aging. 2020;9(1):4-8.

38. Ng TP, Lu Y, Choo RWM, Tan CTY, Nyunt MSZ, Gao Q, et al. Dysregulated homeostatic pathways in sarcopenia among frail older adults. Aging Cell. 2018;(August):1-6.

\section{Figures}




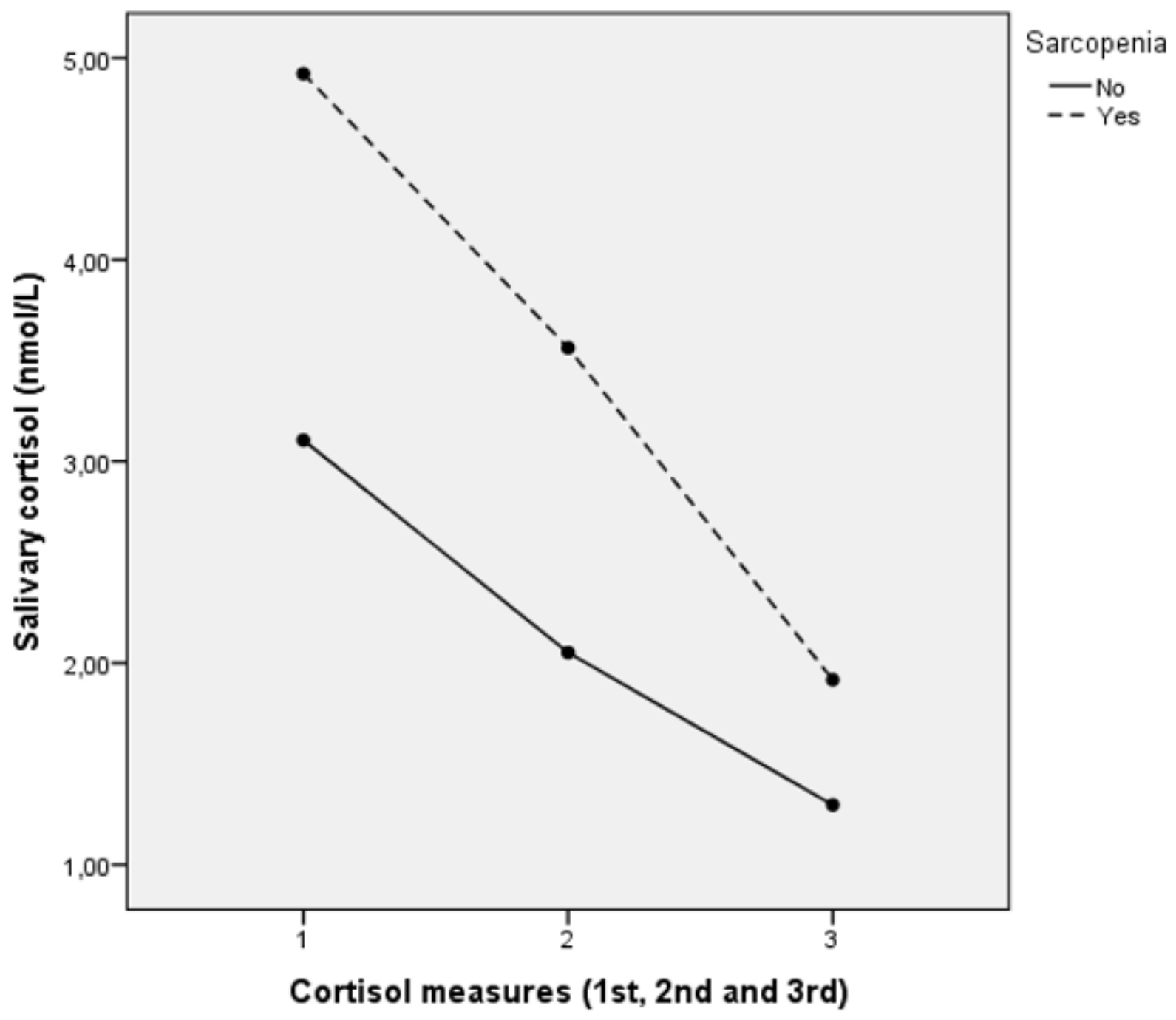

Figure 1

Cortisol levels in an older adult sample in relation to sarcopenia 